\title{
Historia de la radio universitaria: estudios de caso de las emisoras LAUD y UN Radio*
}

\section{University Radio History: Case Studies from the LAUD and the UN Radio Stations*}

\section{Historia da radio universitária: estudos de caso da emissoras LAUD e UM Radio*}

\section{Óscar Julián Cuesta Moreno** • Colombia}

Recibido el 27 de julio de 2012, aceptado el 28 de noviembre de 2012

* Este artículo de investigación hace parte del proyecto sobre la historia de la radio universitaria de la Red de Radio Universitaria de Colombia (RRUC).

** Comunicador Social de la Universidad Santo Tomás. Magíster en educación de la Universidad Pedagógica de Colombia. Especialista en docencia universitaria de la Universidad Cooperativa de Colombia. Técnico en locución y producción de medios audiovisuales del Colegio Superior de Telecomunicaciones.oscarcuesta@colombia.com 


\section{Resumen}

Objetivo: caracterizar la historia de la emisora LAUD, de la Universidad Distrital Francisco José de Caldas, y la UN Radio, de la Universidad Nacional de Colombia sede Bogotá. Metodología: se realizaron estudios de casos, mediante un análisis de documentos institucionales y el análisis de entrevistas semiestructuradas a funcionarios de las emisoras. Resultados: se encontró que el nacimiento de las dos emisoras se debe a proyectos de individuos más que a iniciativas institucionales. El marco legal vigente para la radiodifusión es una limitación importante para la consecución de recursos que permitan la sostenibilidad de estas radioestaciones. Del mismo modo, se observó que las emisoras nacieron para socializar la actividad de la universidad al público en general. Conclusiones: se pueden apreciar dos rasgos característicos de la función de las emisoras: por un lado, difundir lo que hace la universidad (visibilizar la investigación, la vida académica, la proyección social, etc.), sirviendo como puente entre la sociedad y la institución; por otro lado, la producción y difusión de programas culturales y educativos.

Palabras claves: Radio universitaria, radiodifusión, historia radial.

\section{Abstract}

Objective: to characterize the history of the radio stations LAUD of the Francisco José de Caldas University and the UN Radio of the National University of Colombia located in Bogotá. Methodology: case studies were conducted through an institutional documentation analysis and an analysis of semi structured interviews with radio station employees. Results: both radio stations were born thanks to projects developed by individual interests rather than by institutional initiatives. The current legal framework for broadcasting is a major constraint for getting sustainability resources to these radio stations. Similarly, it was observed that both radio stations were born to broadcast the university activities to general public. Conclusions: two characteristics of the radio stations function can be highlighted. On the one hand, it is spreading the university activities (to display research, academic life, social outreach, etc.), working as a bridge between society and the institution, and, on the other hand, producing and disseminating cultural and educational programs.

Keywords: University radio, broadcasting, radio history. 


\section{Resumo}

Objetivo: caracterizar a historia da emissora LAUD, da Universidade Distrital Francisco José de Caldas, e a Um Radio, da Universidade Nacional de Colombia sede Bogotá. Metodologia: realisaram se estudos de casos, mediante um analise de documentos institucionais e o analise de entrevistas semiestruturadas a funcionários das emissoras. Resultados: Encontrou se que o nascimento das emissoras se deve a projetos de indivíduos mais que iniciativas institucionais. Além, o marco legal vigente para a radiodifusão é uma limitação importante para a consecução de recursos que permitam a sostenibilidade destas radio - estações. Do mesmo modo, observou se que as emissoras nasceram para socializar a atividade da universidade ao público em geral. Conclusões: podem se apreciar dois rasgos característicos da função das emissoras: por um lado, difundir o que faz a universidade (visibilizar a pesquisa, a vida acadêmica, a projeção social, etc.), servindo como ponte entre a sociedade e a instituição; por outro lado, a produção e difusão de programas culturais e educativos.

Palavras chaves: radio universitária, radiodifusão, historia radial. 


\section{Introducción}

Algunos autores han publicado artículos sobre la radio universitaria, como Rincón (1998), Gaviria (2007) y González (2009). No obstante, el conocimiento de las emisoras universitarias en el país está poco sistematizado. Aún más, se puede asegurar que hay una notoria ausencia de la academia nacional por dar cuenta de la radio universitaria como objeto de estudio.

En ese orden de ideas, cualquier iniciativa en esta línea es ponderable, pues satisface un conjunto de inquietudes vigentes en espera de ser indagadas. Una de estas preguntas está enmarcada en la historiografía de las emisoras universitarias, pues, si bien emisoras como la de la Universidad de Antioquia y la Universidad Javeriana cuentan con juiciosos ejercicios historiográficos, el resto de ellas carece de publicaciones que den cuenta de sus trayectorias.

Así, pues, se desconoce la historia de las emisoras y se desconocen los propósitos genealógicos de las radioestaciones, las personas que lucharon por su constitución y las dificultades o benevolencias de las instituciones que las administran. Siguiendo esta línea, está por ser comprobada la aseveración de Gaviria (2007) de que las emisoras universitarias nacieron por iniciativas personales, es decir, no como proyectos institucionales.

Aunque, como ya se dijo, hay muchas preguntas que generan las emisoras universitarias como objeto de estudio, la siguiente es la pregunta puntual del presente trabajo: ¿Cuáles han sido la historia y trayectoria de la emisora LAUD, de la Universidad Distrital Francisco José de Caldas, y UN Radio, de la Universidad Nacional de Colombia sede Bogotá?

\section{Consideraciones preliminares}

Los antecedentes de la radio universitaria de Colombia se remontan a 1933, cuando se fundó la emisora de la Universidad de Antioquia. Desde entonces, con una propuesta independiente y alternativa, las emisoras universitarias han tenido un número significativo de oyentes. Si bien el papel de estas radioestaciones es ponderado, no hay producciones académicas que den a conocer las diferentes variables que intervienen en su dinámica.

En la investigación de antecedentes, se observó la documentación más abundante es la de la emisora de la Universidad Javeriana. Entre estos do100 cumentos, se puede destacar el trabajo de Rincón (1998), que habla de los 
objetivos de la fundación de esta emisora y reflexiona, entre otras cosas, sobre sus contenidos, su música y su técnica. Por su parte, González (2009) hace un estudio de audiencias sobre la emisora entre estudiantes de dicha institución.

De estos trabajos historiográficos se destaca el número 24 de la revista 91.9, editada por la emisora de la Universidad Javeriana. Este ejemplar conmemorativo de los 30 años de Javeriana Estéreo procura dar cuenta de la historia de esta emisora y, en ese marco, trae pequeños artículos que narran brevemente algunos hitos de la historia de las emisoras de las universidades Jorge Tadeo Lozano (HJUT 106.9FM), Distrital (LAUDLAÚD 90.4FM) y Nacional (UN Radio 95.9FM), todas de la ciudad de Bogotá.

En esta misma revista, Gaviria (2007) presenta un escrito que sintetiza varios aspectos de la radio universitaria Colombiana. Destaca, como conclusión, que hay necesidad de investigar sobre las emisoras universitarias, y plantea los siguientes interrogantes:

¿A quién sirve la radio universitaria, a quiénes quiere servir y cómo puede servir mejor en el futuro? ¿Cómo medir el impacto de la radio universitaria? ¿Cómo lograr una sostenibilidad para su proyecto comunicacional? ¿Cuál debe ser el rol de la radio universitaria dentro la radio colombiana? ¿Cómo se diferencia la radio universitaria de las demás? (Gaviria, 2007, p. 11)

Si bien Gaviria plantea preguntas sobre la constitución y función de las emisoras, omite indagar respecto a su origen. Sin embargo, al comienzo de su escrito, el autor asegura que "la mayoría de estas emisoras han nacido no como iniciativas institucionales sino como resultado de proyectos individuales, que luego fueron acogidos por las universidades" (Gaviria, 2007, p. 9). Esta última afirmación, que a primera vista parece justa, es necesario convertirla en hipótesis de trabajo y corroborarla en una investigación factual. Una tarea que hasta el momento no ha sido asumida por nadie y que es la principal razón de realizar una investigación que decuenta de la historia y trayectoria de la radio universitaria en Colombia.

Gaviria (2007), por otro lado, se refiere a la tensión legal que da pie a las emisoras universitaria, pues el marco normativo nacional plantea unas tipologías que no satisfacen en su totalidad la razón del ser de las emisoras universitarias. Sin duda alguna, este es otro elemento que es preciso tener en cuenta en una investigación sobre el origen de estas radioestaciones.

En 2003, en el marco del Primer Encuentro Nacional de Radios Ciudadanas y Universitarias, se conformó la Red de Radio Universitaria de Colombia 
(RRUC), organización que ha coordinado proyectos conjuntos y sistematizar el conocimiento de las radioestaciones del país.

Se puede también destacar el trabajo de IESALC y ASCUN (2004), en el que participaron varios investigadores, puesto que estas instituciones realizan una evaluación del estado de los medios de comunicación universitarios. El informe asegura que entre 2000 y 2004 se fundan el mayor número de emisoras universitarias. Además, la mayoría de los medios radiales que participaron en el estudio tienen fuerte dependencia de la institución que los fundó.

Otro trabajo que ha abordado el tema de la radio universitaria es el de Marín (2003), que presentó un ejercicio de una lectura de las parrillas de programación. En otros países, cabe destacar el trabajo de Aguaded y Contreras (2011), coordinadores de un trabajo sobre la radio universitaria como un servicio público en la construcción de una ciudadanía democrática.

\section{Metodología}

Para caracterizar la historia y trayectoria de la emisora LAUD, de la Universidad Distrital Francisco José de Caldas, y UN Radio, de la Universidad Nacional de Colombia sede Bogotá, se siguió un enfoque interpretativo y se utilizó la técnica de estudio de caso. Así, en un primer momento, se recopilaron y analizaron documentos institucionales de las emisoras y, posteriormente, se aplicaron entrevistas semiestructuras. Entre otros, los criterios para seleccionar a los entrevistados fueron: trabajar en las emisoras por períodos mayores a 5 años, estar en los cuadros administrativos y tener acceso a los documentos institucionales.

Las siguientes personas fueron entrevistadas: de LAUD, Alfredo Ardila Godoy (Director), y Alfred Miranda (del equipo administrativo). Por su parte, del equipo de UN Radio se entrevistó a Carlos Emilio Raigoso (Jefe Oficina RadioUnimedios), Pedro Salazar (Realizador y encargado de la fonoteca), Edgar Cuesta (Productor), Carolina Fernández (Coordinadora) y Carlos Fabián Rodríguez (web máster).

\section{Resultados}

Para poner en común los resultados alcanzados, se expondrá, en un primer momento, la descripción del proceso de creación y consolidación de la emisora LAUD, de la Universidad Distrital Francisco José de Caldas y, posteriormente,

102 el caso de la UN Radio de la Universidad Nacional de Colombia sede Bogotá. 


\section{LAUD 90.4.FM}

El Ministerio de Comunicaciones le otorgó a la Universidad Distrital Francisco José de Caldas, mediante la Resolución No. 003430 del 17 de diciembre de 1999, la licencia de servicio de radiodifusión sonora en la frecuencia 90.4 F.M.

Esta licencia, según se puede ver en el Acuerdo 02 de 2002 del Consejo Superior Universitario, compromete a la institución con la ciudadanía, y por ello debe garantizar "la divulgación de programas educativos y culturales".

El artículo 1 del citado Acuerdo es el antecedente formal que da origen a la radioestación, pues resuelve "Crear la emisora de la Universidad Distrital Francisco José de Caldas, con denominación LAUD Estéreo, adscrita a la Vicerrectoría Académica de la Universidad”. Sin embargo, como se pudo ver en las entrevistas, la emisora tiene antecedentes en la iniciativa de un grupo de estudiantes de la facultad de ingeniería electrónica que, a mediados de la década de los ochenta, en la semana institucional de ingeniería, montaron una emisora por los tres días que duraba el evento.

Para el montaje, Caracol prestó algunos equipos y un transmisor para poder emitir desde la sede la Macarena, en la calle 26 con carrera tercera. Si bien la iniciativa tenía la perspectiva de ingenieros del campo electrónico, su inquietud permitió sentar bases más amplias.

"Nos reunimos un grupo en 1995. Cuatro personas generamos el proyecto. Le dijimos a la Universidad la viabilidad de tener la emisora, al rector le gusto y fue cuando se envió la carta al Ministerio. En el transcurso del tiempo, en esos cinco años presentamos la iniciativa, se crearon parrillas, se hicieron estudios. Desde que arrancó la emisora, estoy al frente de la dirección de la emisora" (Alfredo Ardila, entrevista).

Así, a mediados de la década del noventa, el rector de ese entonces, Lombardo Rodríguez López, solicitó al Ministerio de Comunicaciones que le entregara a la Universidad Distrital Francisco José de Caldas una emisora en gestión directa. El Ministerio estudió la posibilidad y, después de dos años, le dio a la Universidad la concesión de la frecuencia 90.4 Fm. La institución tomó tres años aproximadamente para organizar y efectuar la comprar de los equipos y hacer estudios técnicos, jurídicos y administrativos.

Solo hasta el 10 de noviembre de 2000 a las 12:00m salió al aire LAUD 90.4 FM, en el salón Gonzalo Jiménez de Quesada de la Alcaldía Mayor de Bogotá. Actualmente, la emisora funciona con 24 horas de programación continua." (Alcaldía de Bogotá, 2006). [En detalle]: "La primera transmisión se 
hizo un medio día desde la Secretaría de Gobierno del Distrito y después de escucharse las notas del Himno de Colombia y de Bogotá (Alcaldía de Bogotá, 2004).

Como se puede ver, si bien LAUD en 2012 cumple 12 años, sus antecedentes tienen casi 30 años. Aquí, se puede ver claramente la idea expuesta por Gaviria (2007) de que las emisoras tienen origen en compromisos individuales que después de sortear límites, han sido apoyados por las instituciones. En las entrevistas se encontró que, en el caso de esta emisora, el compromiso de Alfredo Ardila fue fundamental.

Entre los cuestionamientos que se hicieron en su momento, llama la atención que se pusiera en duda el proyecto de la emisora porque la Universidad Distrital no tenía una facultad o un programa de comunicación, periodismo o similares.

Por otro lado, antes de salir al aire LAUD, el equipo de trabajo observó la oferta de otras emisoras universitarias de Bogotá (la Distrital fue la última emisora en aparecer de las cuatro estaciones universitarias presentes en Bogotá; las otras tres son Universidad Javeriana, Universidad Nacional y la Jorge Tadeo Lozano). Por ello, procuraron no imitar sus estilos. Por eso le dieron prioridad a la opinión de los estudiantes, para que ellos les dieran más opciones.

Así, en el desarrollo de la parrilla de programación, jugó un papel importante la interacción con los estudiantes y los docentes de la institución. Tras consultar a estos grupos, la emisora procuró evitar el esquema de música clásica: "Salimos de ese esquema de todas las emisoras universitarias y decidimos armar nuestra propia programación musical" (Alfredo Ardila Godoy, comunicación personal, 15 de junio 2012). La novedad radicó en darle preeminencia a la música latinoamericana y, en esa línea, música no es comercial.

El crecimiento de LAUD se puede ver en el aumento de su personal: de cinco personas que componían el equipo en 2000. Después de 12 años, la planta entre técnicos, periodistas, locutores y administrativos asciende a 22 personas. Del mismo modo, las transformaciones en estos años se ven en la formulación de contenidos, pues los entrevistados manifestaron que comenzaron con programas musicales y, con el paso del tiempo, se dio la necesidad de crear espacios formativos y culturales, intercalados con música folclórica. Esa necesidad emergió del intercambio con los oyentes.

En la búsqueda de información, algunos hechos significativos de la emisora han sido: 
- En vísperas de celebrar su cuarto aniversario, el 30 de octubre de 2004, un rayo causó daños en el transmisor y dejó a la emisora fuera del aire durante cinco días. El incidente "ratificó a sus periodistas, locutores y productores, gracias a las llamadas y a los correos electrónicos, que es una emisora que acompaña no sólo a los universitarios de la Distrital, sino, además, a un amplio sector de Bogotá” (Alcaldía de Bogotá, 2004).

- El convenio con Radio Francia Internacional que en 2012 cumplió cinco años.

- En 2005, se realizó la lectura ininterrumpida del libro El ingenioso hidalgo Don Quijote de la Mancha, celebrando así los 400 años de este clásico de la literatura. "La actividad contó con la participación 1071 ciudadanos de todas las localidades, colegios, universidades y figuras del ámbito político, cultural e intelectual. En esta forma, se logró un record mundial de lectura luego de leer página a página, persona a persona en vivo durante 39 horas de transmisión continua" (Universia, 2006).

- En 2006, con seis años de existencia, lo que es poco tiempo comparado con las otras emisoras universitarias de Bogotá, se registraban "28.200 oyentes en el universo de escuchas de radios universitarias que es de 150 mil oyentes en la ciudad" (Universia, 2006). En esta forma, se observa un crecimiento significativo.

- Para celebrar su primera década, se realizó el Concierto LAUD Estéreo 90.4 Fm, 10 Años de Verdadera Alternativa, en el tradicional teatro al aire libre la Media Torta en el centro de Bogotá (Distrito Capital Radio, 2010).

- En su parrilla de programación se resaltan programas como "Música y músicos de Colombia", "Revista de la mañana" y "Amantes del círculo polar", que se han transmitido desde el inicio de la emisora (Libros y Letras, 2010).

- Según el director, Alfredo Ardila Godoy, son la única emisora universitaria en Colombia con móvil para transmitir en directo. La unidad móvil se lleva a colegios distritales para mostrarle a los estudiantes cómo es la dinámica de la producción radiofónica y, al mismo tiempo, discutir sobre los problemas de la localidad con ellos. Entre otros, se destacan los cubrimientos de Rock al Parque.

La emisora afirma que su misión es "difundir la cultura, la ciencia y la tecnología en Bogotá (...) Además, ser puente de interacción entre la Universidad y el entorno social (Comunidad, Entidades centralizadas y descentralizadas, ONG, etc.), cumpliendo con el objetivo social que la Academia persigue" 
(LAUD, 2012). Por otro lado, las personas entrevistadas hicieron hincapié en que la emisora sirve para visualizar la Universidad, esto es, socializar la vida académica de la institución.

Para la difusión de lo que hace la Universidad en ciencia, tecnología e investigación, hay programas especializados realizados por los periodistas de la emisora con el acompañamiento de los profesores. Cada facultad de la Universidad tiene un programa y lo utilizan según sus criterios, con participación de estudiantes y docentes.

Es ponderable que LAUD ha realizado capacitaciones a personas de diferentes localidades de Bogotá en la gestión y creación de distintos medios alternativos en prensa, radio, televisión y la internet, con el objetivo de que los ciudadanos cuenten con herramientas para construir sus propios medios de comunicación. Se puede leer en una nota que "La capacitación se ha realizado durante los últimos tres años a más de 1.200 líderes comunitarios, indígenas, negritudes y estudiantes" (Alcaldía de Bogotá, 2006).

La emisora ha tenido un fuerte vínculo con diferentes secretarías de la Alcaldía Mayor de Bogotá, dado que la Universidad Distrital Francisco José de Caldas es la institución pública de educación superior de esta ciudad. Sin embargo, cabe decir que en las entrevistas se enfatizó que la emisora no recibe ninguna presión de la administración de la Universidad ni de ninguna dependencia de la Alcaldía.

Con respecto a los desarrollos tecnológicos de la emisora, los entrevistados destacan el papel de la página web de la estación (www.laud.distrital.edu.co), pues permite la interacción con los docentes, estudiantes y público en general y logra la difusión de noticias y novedades de la Universidad.

Respecto a los problemas más reiterados en los 12 años de historia de la emisora, se deben destacar las limitaciones financieras por causa de la figura legal que enmarca a las emisoras universitarias.

En efecto, tal como lo indica Gaviria (2007), el marco legal que reglamenta la radiodifusión en Colombia clasifica a las emisoras universitarias como Radiodifusión sonora de interés público. Puntualmente, la normatividad describe a estas emisoras de la siguiente manera:

Cuando la programación se orienta a satisfacer necesidades de comunicación del Estado con los ciudadanos y comunidades, la defensa de los derechos constitucionales, la protección del patrimonio cultural y natural de la nación, a fin de procurar el bienestar general y el mejoramiento de la calidad

Para $\quad$ Cuesta M., Óscar Julián Historia de la radio universitaria: estudios de caso de la emisora 
de vida de la población, sin ánimo de lucro, a cargo y bajo la titularidad del Estado (Artículo 18 del Decreto 2805 de 2008).

La limitación estipulada en el decreto radica en que los servicios de radiodifusión sonora de interés público:

No se podrá transmitir pauta comercial, salvo los patrocinios entendidos como el reconocimiento, sin lema o agregado alguno, a la contribución en dinero u otros recursos en favor de las emisoras de interés público que se efectúen para la transmisión de un programa específico y sobre el cual podrá hacerse un reconocimiento no superior a cinco (5) minutos por hora de programación del programa beneficiado. La institución pública que solicite la licencia para una emisora de interés público debe garantizar su sostenibilidad técnica, de contenido, administrativa y financiera (Artículo 25 del Decreto 2805 de 2008).

Así, en LAUD, el dinero proviene de campañas del Distrito (administración pública de Bogotá) o del Estado. Los entrevistados destacaron la necesidad de modificar ciertos aspectos de la norma para ampliar los criterios de patrocinio y aumentar las posibilidades de ingreso de la emisora. El director manifestó que varias veces instituciones privadas han querido pautar (pasar cuñas comerciales), pero él no ha podido aceptar los contratos por los límites legales.

El artículo 11 del Acuerdo 02 de 2002 del Consejo Superior Universitario estipula que los recursos de la emisora provienen de "aportes, colaboraciones, auspicios, patrocinios que reciba directamente de instituciones públicas o privadas o personas naturales y el presupuesto asignado por la Universidad y los derivados de la Prestación de Servicios". Es decir, que LAUD tiene dos tipos de ingresos: el presupuesto que le asigne la Universidad Distrital y la gestión que la radioestación realiza.

Respecto a sus proyecciones, el equipo de la emisora coincide en que planea hacer un mejor uso de las posibilidades que brinda la internet para la radio (la página web de la emisora se creó hace tres años). Esto implica reconocer la posibilidad de oyentes alrededor del mundo y, por supuesto, da lugar a una programación interactiva y global.

\section{UN Radio 95.9.FM}

Los antecedentes legales de la creación de UN Radio se encuentran en el Decreto $\mathrm{N}^{\circ} 2000$ del 24 de junio de 1986, que permite a la Universidad Nacional acceder a la "Creación de órganos de comunicación masiva que le permitan un contacto permanente y eficaz con la sociedad". 
La Radio Universidad Nacional, primer nombre de UN Radio, se creó oficialmente bajo el Acuerdo No 126 de 1986 del Consejo Superior Universitario de la Universidad Nacional de Colombia. Por su parte, la Resolución Nº 160 de 1986, emanada del mismo organismo, autoriza al Rector a firmar un contrato con el Ministerio de Comunicación para una concesión de Radiodifusión. En ésta se puede leer: "Es importante que la Universidad Nacional disponga de los medios necesarios para la difusión de sus actividades docentes, científicas y culturales".

Con estas bases, el 17 de noviembre de 1987, se firma el contrato 0453 del Ministerio de Comunicaciones con la Universidad Nacional de Colombia. Fue firmado en ese entonces por Fernando Cepeda Ulloa, Ministro de Comunicaciones, y Marco A. Palacios Rozo, Rector de la Universidad Nacional de Colombia.

En este documento, se establece que la emisora tendrá la Frecuencia Modulada (FM) $97.4 \mathrm{MHz}$; su distintivo de llamada será H.J.Y.V.; su potencia es de 1 Kilovatio en antena; su frecuencia de enlace es de $314.1 \mathrm{MHz}$; y su ubicación será en las Residencias Uriel Gutiérrez de la Universidad Nacional de la ciudad de Bogotá. El objeto del contrato es "difundir la verdad y elevar el nivel cultural y la salud de la población, preservar y enaltecer las tradiciones nacionales"; además, favorecer la democracia, la paz nacional y la cooperación internacional. Así mismo, se reservan dos horas diarias para realizar programas de educación a distancia. Mediante la Resolución Número 005201 de 1987 de la Dirección Nacional del Derecho de Autor quedó reservado el nombre Radio Universidad Nacional.

Los antecedentes de producción de UN Radio se remontan a los ejercicios de profesores que grababan audios de temas académicos y eran transmitidos por la Radio Nacional de Colombia, la estación estatal. La emisora fue inaugurada formalmente en 1991, con la dirección de Fernando Orjuela.

Años después, en 1997, la emisora quedó articulada a una oficina de comunicaciones y medios que es la Unidad de Medios de Comunicación -Unimedios-. La Resolución 334 de 2007 de la Rectoría de la Universidad Nacional de Colombia establece las funciones generales de esta dependencia. Entre otras, se puede destacar la de hacer públicas las actividades de la universidad y "producir elementos de análisis de la información y de los medios de comunicación que le permitan a la Universidad Nacional de Colombia observar las transformaciones sociales nacionales e internacionales que afectan la vida universitaria" (p.5). 
La Oficina de radio queda, pues, adscrita a UNIMEDIOS y tiene, entre otras, las siguientes funciones:

Establecer, producir y transmitir programación radial 24 horas del día, que promueva la educación, la cultura y la ciencia que se producen en la Universidad Nacional de Colombia (...) Producir y transmitir programas radiales educativos y culturales que promuevan la radio universitaria como alternativa cultural a nivel nacional y regional (Rectoría Universidad Nacional, 2007, p. 5).

Para Raigoso (2007), "La idea de crear una emisora suena desde los sesenta, pero llega a concretarse solo hasta los noventa, como un proyecto de ingeniería más que de comunicaciones" (p. 23). Respecto a la línea de programación, se puede ver que la emisora de la Universidad Nacional tomó como referente la Radiodifusora Nacional, radio-estación pública de corte cultural:

Nosotros tomamos más la línea de la Radiodifusora y la hicimos fuerte. Propusimos una emisora muy discursiva, aunque había unas franjas grandes de música. Queríamos mostrar todo lo que se hacía en la universidad, ¡tanto que algunos programas, incluso, parecían clases! Esa fue una primera propuesta que fue variando con cada director (Raigoso, 2007, p. 23).

"El 22 de septiembre 1991 la emisora salió al aire con una programación oficial, musical y académica; en ese momento estábamos 8 horas diarias de lunes a viernes. Más adelante se pasó a 18 horas y, más o menos en 1993 fueron 24 horas" (Pedro Salazar, entrevista). La emisora de la universidad en la sede de Medellín (100.4 FM) nació una década después.

De la misma manera que la Universidad Distrital, la Universidad Nacional no cuenta con un programa o facultad de Comunicación Cocial o de Periodismo. Solo hace poco se abrió una maestría en esta línea. Tal parece que la ausencia de programas en comunicación y medios fue una dificultad al conformar la emisora, pues, uno de los entrevistados señaló que no se contaba con personal profesional en la producción radiofónica. Esa falencia se solucionó con el compromiso de las personas y directivos que creían en el proyecto.

La 98.5 UN Radio y su programación son el resultado de la relación entre experticia y comunicación. En primer lugar, es el trabajo de ingenieros y técnicos que lograron establecer un soporte tecnológico adecuado para el desarrollo de un proyecto de comunicación. Ellos son los garantes de la calidad y la permanencia de la señal (Raigoso, 2006).

El personal que llegó a trabajar en la naciente emisora provenía de la Radio Difusora Nacional y de otra emisora cultural llamada Musicar Estéreo, de allí que el proyecto tuviera la influencia del formato radiofónico culturalista. 
Las modificaciones en las dinámicas de producción en su historia presentan renovaciones en los equipos y en el software de producción, a los que el personal técnico se ha ido adaptando (Universia, 2007). Sin embargo, a lo largo de los años hay una constante en la dinámica de producción: la disposición articulada de profesores, estudiantes y comunicadores para la construcción de espacios y contenidos.

La propuesta musical de la emisora se ha modificado progresivamente. En un principio, siguiendo la línea de radio-estaciones culturales, se privilegiaba la música clásica. Posteriormente, se dio pie al rock y a la música colombiana. Esta última tenía un espacio de una hora a la semana, hoy es de 8 horas. Cada semana se emite música de las distintas regiones y de los distintos géneros nacionales.

Igualmente, se pueden ver esas progresiones en los contenidos hablados. En un principio, los espacios en los que se privilegia la palabra no pasan de dos horas diarias, en la actual producción es alrededor del 45 por ciento de la programación. De hecho, la discusión y el análisis se ha convertido en un rasgo característico de la emisora y los oyentes bogotanos la sintonizan por permitir la construcción de opinión (UN Periódico, 2010).

También se articula el trabajo realizado por la estación de Bogotá y su par en Medellín. Según Carolina Fernández, coordinadora de la emisora bogotana, se realizan algunos intercambios de material radiofónico y se procura saber qué se está trasmitiendo en cada una de las emisoras.

Se pudo determinar que algunos hechos significativos de la emisora han sido:

- En la parrilla de programación, los entrevistados destacan dos programas: por un lado, la franja de rock, que pasa de lunes a viernes de 11 a 12 de la noche, pues va a cumplir 20 años. Por otro lado, UN Análisis, que es un programa académico e informativo en el que se debaten temas de coyuntura.

- Respecto al aporte que realiza la emisora a las funciones sustantivas de la universidad, su director señala que la mayor contribución la realiza en la investigación, en la socialización de los resultados obtenidos por los proyectos ejecutados en la institución. Indica que esto es muy significativo, pues, siendo una universidad financiada por dineros públicos, es democrático difundir cómo y en qué se invierten los dineros del presupuesto y la relevancia de estas investigaciones para los problemas colombianos.

"Yo recuerdo que uno de los primeros directores decía que todo lo que saliera por la emisora de la Universidad Nacional, siendo el centro de conocimiento y de investigación más importante del país, tenía que salir impregnado de 
algún tipo de contenido, es decir, si yo hablaba de música tenía que tener en cuenta la música en sus detalles" (Carlos Emilio Raigoso, entrevista).

- Entre los muchos invitados al estudio, se recuerda al lingüista Noam Chomsky.

- La emisora ha ganado alrededor de 17 reconocimientos, entre ellos, varios Simón Bolívar (máximo premio colombiano para periodistas), un premio de radio educación en México y varios premios del Círculo de Periodistas de Bogotá.

- De los directores de la emisora, los que más se recuerdan son Héctor Martínez, Fernando Orjuela, Olga Marín y Fabio Fandiño porque sus orientaciones dejaron huella en la identidad de la emisora. Según Raigoso (2007), para la historia de la emisora fue muy importante la dirección de Olga Marín, en 1997, que encaminó sus esfuerzos a eliminar la barrera entre los comunicadores y los académicos, logrando que estos fueran más comunicativos y aquellos más rigurosos.

- Para celebrar sus 15 años al aire, la UN Radio realizó un concierto el 22 de septiembre de 2006 en la concha acústica del campus de la Sede Bogotá (Universia, 2006).

- De manera anecdótica, al día siguiente de cumplir 20 años, el 23 de septiembre de 2011, uno de los programas emblemáticos de la emisora, UN Análisis, recibió un premio de responsabilidad ambiental.

Respecto a los problemas, las entrevistas registran que el más significativo es el marco legal que rige a la UN Radio, específicamente, la normatividad que no permite a las estaciones de interés público recibir pautas comerciales (Decreto 2805, 2008).

Otro problema señalado son las modificaciones en la dirección de la universidad, pues hay antecedentes de que la nueva administración hace cambios en el personal de la emisora y esto tiene consecuencias en la programación porque produce rupturas, no transiciones.

La primera página web de UN Radio apareció cuando la emisora cumplió nueve años, pero fue una propuesta solamente informativa, no tenía una concepción de las posibilidades de la multimedia. Desde 2005, se comenzaron a incluir algunos audios en ella. Actualmente, la página tiene todo lo que transmite la emisora y existe una emisora web con contenido propio.

Otro aspecto relevante es la dinámica de redes sociales de la emisora. En la cuenta de Facebook, se pueden encontrar los avances de los programas de 
la emisora y el contenido textual que se va a escuchar en el programa. La web, según dicen los entrevistados, permite atraer una cantidad de oyentes nuevos y no solamente de Bogotá o del país.

Una de las perspectivas futuras de la UN Radio sería ampliar sus servicios en internet, lo que hace posible tener producción propia de las diferentes sedes de la Universidad Nacional, como San Andrés, Leticia o Arauca, y satisfacer así las necesidades locales de contenido.

\section{Conclusiones}

Al hacer una comparación de la trayectoria de las dos emisoras, se puede encontrar que desde la iniciativa hasta la primera emisión hay varios años. En la gestación de LAUD se pueden identificar dos momentos desde 1995 (comienzo de las gestiones) a 2000 (primera emisión): una primera etapa de justificación que termina con la solicitud del rector al Ministerio de Comunicación (1997). Una segunda etapa de consolidación, que comienza con la aprobación del ministerio, transcurre con la compra de equipos y el diseño de parrillas de programación y termina con la primera emisión.

Por su parte, en la vida de la UN Radio se pueden ver tres momentos: el primero es a finales de los ochenta cuando se crean los estudios y se comienzan a grabar allí programas que eran transmitidos por la Radiodifusora Nacional (bajo la dirección de Héctor Martínez). Posteriormente, se inicia el proceso legal con el Ministerio (1987) en el que se asigna la frecuencia. Finalmente, el nacimiento con la primera emisión formal en 1991 (director Fernando Orjuela).

Igualmente, en los dos casos, el afán por el proyecto radiofónico tiene origen en inquietudes formuladas por la ingeniería. En ese sentido, las ideas que dieron vida a LAUD y a UN Radio fueron puestas en duda porque las universidades Distrital y Nacional no tenían facultades o programas de comunicación, periodismo o similares.

Desde sus inicios hasta hoy, se pueden ver dos rasgos característicos de la función de las emisoras: por un lado, difundir lo que hace la universidad (visibilizar la investigación, la vida académica, la proyección social, etc.), para servir de puente entre la sociedad y la institución; por otro lado, la producción y difusión de programas culturales y educativos.

En cuanto a su organigrama, desde el inicio las dos radioestaciones están

112 adscritas a los cuadros rectorales. LAUD a la Vicerrectoría Académica y en UN 
Radio la rectoría directamente (hace parte de UNIMEDIOS, pero este organismo está dirigido por el rector). En los dos casos, las normas que regulan el funcionamiento de las emisoras están estipuladas por documentos emanados del Consejo Superior Universitario.

Es ponderable que en la trayectoria de las dos emisoras estudiadas se de la participación de estudiantes y docentes en la producción de programas. Del mismo modo, se escucha cada vez más una sinergia entre la labor del profesor (especialista en un tema) y los comunicadores sociales que trabajan en la emisora.

En los dos casos, al indagar sobre los proyectos futuros, los entrevistados centraron las perspectivas de las emisoras en las posibilidades que presenta la web para la radio, como la presentación de contenidos multimediales y la interacción con oyentes en todo el mundo.

Por otro lado, hay varias diferencias entre las dos emisoras en el campo de la programación y los contenidos radiofónicos ofrecidos. En un principio, UN Radio tuvo como referente de contenidos dos emisoras de corte cultural (como la música clásica), pero después fue buscando otra identidad. Por su parte, LAUD, al ser la emisora universitaria más joven de la ciudad de Bogotá, pudo marcar diferencias que le permitieran tener un rasgo diferenciador (como la música latinoamericana). En ese sentido, se puede afirmar que los rasgos que definen los parámetros identitarios de las emisoras estudiadas están en permanente renovación.

En relación con la historia y la trayectoria de las emisoras, uno de los mayores contrastes es la continuidad en la dirección de la emisora: LAUD ha sido orientada por un solo director en sus 12 años de existencia; la UN Radio, por el contrario, ha tenido varios directores, lo que según las entrevistas ha dado lugar a rupturas, más que transiciones.

Se observó que las genealogías de las dos emisoras estudiadas tienen raíces en empresas personales que, gracias al compromiso de sus gestores, alcanzaron luego apoyo institucional. En ese sentido, se ratifica la hipótesis de Gaviria (2007) de que las radioestaciones universitarias no nacieron como proyectos institucionales sino como iniciativas defendidas por personas.

De igual modo, se ratificó que se da una tensión legal en el marco que cobija a las emisoras universitarias, dado que la tipología y la normatividad planteada en el Decreto 2805 de 2008 no satisfacen en su totalidad la razón del ser de las emisoras universitarias y, de manera significativa, limita sus posibilidades de ingresos lo que repercute en su sostenibilidad. 
El caso de LAUD (fundada en 2000) permite ver una de las conclusiones del trabajo realizado por IESALC y ASCUN (2004), quienes aseguran que el mayor número de emisoras universitarias fundadas en Colombia ocurre entre 2000 y 2004. Contrario a UN Radio, que se fundó en 1991.

Los resultados presentados aquí, igualmente, permiten corroborar otra de las conclusiones presentadas por IESALC y ASCUN (2004), pues se demuestra que las emisoras radiales universitarias estudiadas tienen una fuerte dependencia institucional. Esto, entre otras cosas, se debe al marco legal que limita sus ingresos a los presupuestos asignados por la universidad, pues no tienen la posibilidad de tener pautas comerciales.

Finalmente, es necesario seguir indagando sobre la historia de las emisoras universitarias en Colombia, aún más cuando gran parte de ellas emite sus contenidos en web porque no tienen concesión de frecuencias. Así mismo, siguen abiertas las inquietudes planteadas por Gaviria (2007), especialmente aquellas que invitan a indagar sobre el rol de la radio universitaria en Colombia y las diferencias de la radio universitaria con respecto a las otras emisoras.

\section{Referencias}

Alcaldía de Bogotá (2006). Emisora del Distrito cumple 6 años de estar al aire. Bogotá: Alcaldía de Bogotá.

Alcaldía de Bogotá (2004). Los cuatro años de LAUD 90.4 F.M Estéreo. Bogotá: Alcaldía de Bogotá.

Decreto 2805 (2008). Diario Oficial No. 47.067 de 31 de julio.

Distrito Capital Radio (2010). Concierto de diez años de verdadera alternativa de LAUD. Bogotá: Alcaldía de Bogotá.

PUJ (2009). Estudio de audiencias a estudiantes de la Pontificia universidad Javeriana en relación a la emisora Javeriana Estéreo. Bogotá: Pontificia Universidad Javeriana.

Gaviria (2007). La radio universitaria en Colombia. Revista 91.9 La revista que suena, (24) 9-11.

IESALC y ASCUN (2004). Medios de comunicación universitarios en Colombia. Situación actual y perspectivas. Bogotá: Asociación Colombiana de Universidades

UN (200x). La 98.5 FM Una radio de 15 años. UN Periódico No. 982006

Para 
La radio en la Universidad Javeriana (1998). El Hilo de Ariadna 2.

La radio universitaria: Como servicio público para una ciudadanía democrática2011La CoruñaNetbiblo

LAUD (2012). Quiénes somos. Bogotá: Universidad Distrital Francisco José de Caldas.

MdeC (2003). Lectura de Parrillas Emisoras AM y FM. Bucaramanga: Ministerio de Cultura.

Libros y Letras (2010). La emisora Distrital Francisco José de Caldas celebra sus 10 años. Bogotá: Universidad Distrital Francisco José de Caldad.

UN (2007). Resolución 334. Bogotá: Universidad Nacional.

UN Periódico (2010). UN Radio (98.5 FM) se consolida entre los líderes de opinión. UN periódico.

UN RADIO 95.9 FM (2007). 91.9 La revista que suena (24), Noviembre 23.

Universia (2006). LAUD 90.4 FM: seis años colocando a Latinoamérica en su corazón.

Universia (2007). Modernización en la Nacional: la señal de UN Radio se volverá digital.

Universia (2006). UN Radio celebra sus 15 años cargada de música.

Para $\quad$ Cuesta M., Óscar Julián Historia de la radio universitaria: estudios de caso de la emisora 